\title{
The F/D Ratio and Meson-Baryon Couplings from QCD Sum Rules
}

\author{
Takumi Doi ${ }^{\mathrm{a} *}$, Hungchong Kim ${ }^{\mathrm{b}}$, Yoshihiko Kondo ${ }^{\mathrm{c}}$ and Makoto Oka ${ }^{\mathrm{a}}$

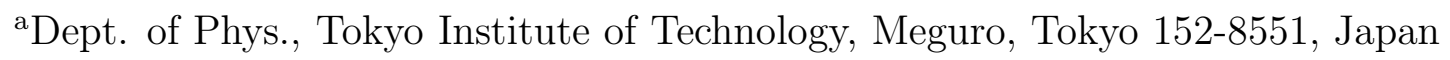 \\ bInst. of Phys. and Applied Phys., Yonsei University, Seoul 120-749, Korea \\ 'Kokugakuin University, Higashi Shibuya, Tokyo 150-8440, Japan
}

\begin{abstract}
Coupling constants of the pseudoscalar mesons to the octet baryons are computed in the QCD sum rule approach. The $\pi N N, \eta N N, \pi \Xi \Xi, \eta \Xi \Xi, \pi \Sigma \Sigma, \eta \Sigma \Sigma$ as well as $\pi \Lambda \Sigma$ couplings are studied. Determining the pertinent Dirac structure in the correlation function, we analyze the couplings in the $\mathrm{SU}(3)$ limit. We find the F/D ratio to be $\sim 0.6-0.8$ that is consistent with the $\mathrm{SU}(6)$ value. We also estimate the $\mathrm{SU}(3)$ breaking effect using a projected correlation function method.
\end{abstract}

\section{INTRODUCTION}

Recent developments in hypernuclear physics require us knowledges of the interactions among strange hadrons, such as hyperons $(\Lambda, \Sigma, \ldots)$ and kaons. Much efforts have been made to analyze experimental data to draw the consistent picture of the hyperon interactions. Yet the most fundamental quantities, i.e., the meson-baryon coupling constants, which are essential in constructing the meson exchange forces of baryons, have been treated as free parameters. There the SU(3) invariance of the coupling constants is often employed without foundation. Under these circumstances, it is highly desirable to calculate the meson-baryon coupling constants from the fundamental theory.

In this report, we present a QCD sum rule calculation of the coupling constants of the pseudoscalar mesons to the octet baryons. The QCD sum rule has been successful in determining the masses and couplings of various hadrons in terms of the QCD vacuum condensates and the other fundamental constants. In applying the sum rule technique, several technical points are carefully considered first. The soft pion (and other pseudoscalar mesons) is often used in deriving the sum rule for the coupling constants. We stress that the finite meson momentum should be considered in order to obtain reliable sum rule. We need to choose the Dirac structure of the correlation function carefully so that the sum rule is practically workable. It is also necessary to check dependence on the choice of the interpolating field operator for the baryon. The last point is especially important in deriving the $\mathrm{F} / \mathrm{D}$ ratio for the coupling constants.

In section 2, we present the formulation for the sum rule of baryon-diagonal coupling constants and the results of the Borel sum rule analyses. We derive the $\mathrm{F} / \mathrm{D}$ ratio in the

*E-mail: doi@th.phys.titech.ac.jp 
analysis in the $\mathrm{SU}(3)$ limit. In section 3, we derive the sum rule for the $\pi \Lambda \Sigma$ coupling constant as an example of the baryon-off-diagonal case, where we use the projection method to define the coupling constant unambiguously.

\section{SUM RULE FOR THE MESON-BARYON COUPLING CONSTANTS}

We analyze baryon-diagonal meson-baryon couplings $\mathcal{M} B$ for meson $\mathcal{M}=\pi, \eta$ and baryon $B=N, \Xi, \Sigma$. We construct QCD sum rules from the two-point correlation function with an external meson field

$\Pi(q, p)=i \int d^{4} x e^{i q x}\left\langle 0\left|\mathrm{~T}\left[J_{B}(x) \bar{J}_{B}(0)\right]\right| \mathcal{M}(p)\right\rangle$,

where $J_{B}$ is general baryon current without derivative. The explicit formula for $J_{N}$ is $J_{N}(t)=2 \epsilon_{a b c}\left[\left(u_{a}^{T} C d_{b}\right) \gamma_{5} u_{c}+t\left(u_{a}^{T} C \gamma_{5} d_{b}\right) u_{c}\right]$, and $J_{\Xi}$ and $J_{\Sigma}$ are obtained via $\mathrm{SU}(3)$ rotations of $J_{N}$. We note general currents have free mixing parameter $t$, which is essential to check the reliability of sum rules later. It is known that if one takes the soft-meson limit $p \rightarrow 0$, the sum rule from Eq. (1) becomes equivalent to the chiral-rotated nucleon mass sum rule via the soft-meson theorem [1], and reduces to the Goldberger-Treiman relation with $g_{A}=1$ [2]. Therefore, it is necessary to go beyond the soft-meson limit and determine the coupling constants independently of mass sum rule.

When going beyond the soft-meson limit, we have three distinct Dirac structures $\left(i \gamma_{5} \not p\right.$, $i \gamma_{5}$ and $\gamma_{5} \sigma_{\mu \nu} q^{\mu} p^{\nu}$ ) in the correlation function [ 3, 4, 5] as

$\Pi=i \gamma_{5} \not p \Pi_{\mathrm{PV}}+i \gamma_{5} \Pi_{\mathrm{PS}}+\gamma_{5} \sigma^{\mu \nu} q_{\mu} p_{\nu} \Pi_{\mathrm{T}}$.

From each Dirac structure, we obtain sum rules for the coupling constant $g_{\mathcal{M B}}$ as

$g_{\mathcal{M} B} \lambda_{B}^{2}(t)\left[1+A_{\mathcal{M} B}(t) M^{2}\right]=f_{\mathcal{M} B}^{\mathrm{OPE}}\left(M^{2} ; t\right)$,

where $M$ denotes the Borel mass, $\lambda_{B}(t)$ overlap constant between the current and the corresponding baryon, $A_{\mathcal{M B}}$ unknown parameter. We calculate OPE term $f_{\mathcal{M} B}^{\mathrm{OPE}}\left(M^{2} ; t\right)$ up to dimension 8 at $\mathcal{O}\left(p^{2}\right)\left(i \gamma_{5}\right.$ structure), and up to dimension 7 at $\mathcal{O}(p)\left(i \gamma_{5} \not p\right.$ and $\gamma_{5} \sigma_{\mu \nu} q^{\mu} p^{\nu}$ structures) [5]. Considering $m_{q} \sim \mathcal{O}\left(p^{2}\right)$ indicated by the Gell-Mann-OakesRenner relation, we include $\mathcal{O}\left(m_{q}\right)$ terms only in $i \gamma_{5}$ structure. By linearly fitting r.h.s. of Eq. (3) against $M^{2}$, we determine $\left[g_{\mathcal{M B}} \lambda_{B}^{2}(t)\right]_{\text {fitted }}$.

\subsection{Pertinent Dirac structure for coupling sum rules}

In principle, sum rules from any Dirac structure $i \gamma_{5} \not p, i \gamma_{5}$ and $\gamma_{5} \sigma_{\mu \nu} q^{\mu} p^{\nu}$ can be used to determine the couplings. In practice, however, different prediction is derived because reliability of the sum rule varies depending on the Dirac structure. Therefore one must carefully choose pertinent Dirac structure.

First, we point out that the sum rules from $i \gamma_{5} \not p$ structure is highly sensitive to the continuum threshold $S_{0}$ and thus is not reliable. On the other hand, the sum rules from $i \gamma_{5}$ and $\gamma_{5} \sigma_{\mu \nu} q^{\mu} p^{\nu}$ structure are insensitive to $S_{0}$ and more appropriate than those from $i \gamma_{5} \not p$ structure [ 3, 甘, 5].

Second, we check the dependence on the choice of the baryon current. The $t$ dependence in Eq. (3) gives a new constraint. In the SU(3) limit (i.e. $\left.\lambda_{N}=\lambda_{\Xi}=\lambda_{\Sigma}\right), g_{\mathcal{M} B} \lambda_{B}^{2}(t)$ has a common $t$ dependence and thus the ratios of different couplings are $t$ independent. We 
find that the sum rules from $\gamma_{5} \sigma_{\mu \nu} q^{\mu} p^{\nu}$ structure satisfy the above constraint well, while the sum rules from $i \gamma_{5}$ structure do not. Therefore, we conclude that $\gamma_{5} \sigma_{\mu \nu} q^{\mu} p^{\nu}$ structure is the most pertinent Dirac structure for coupling sum rules.

\subsection{Determination of the $F / D$ ratio}

In the $\mathrm{SU}(3)$ limit, we confirm that our sum rules satisfy the $\mathrm{SU}(3)$ relation between couplings. We first determine $\left[g_{\mathcal{M} B} \lambda_{B}^{2}(t)\right]_{\text {fitted }}$ by linear fitting Eq. (国) for every $\mathcal{M}=\pi, \eta$ and $B=N, \Xi, \Sigma$. We calculate the ratio between them and convert it into the $F / D$ ratio. Note that ambiguity of $\lambda_{B}$ cancels because $\lambda_{N}=\lambda_{\Xi}=\lambda_{\Sigma}$ in the SU(3) limit.

In figure 1, we show the $F / D$ ratio against the choice of baryon current. Here, we introduce a new parameter $\theta$, which is defined as $\tan \theta=t$. As discussed before, we confirm that the $F / D$ ratio is insensitive to both of the continuum threshold $S_{0}$ and the parameter $\theta$ in the realistic region $(-0.78 \lesssim \cos \theta \lesssim 0.61)$ [ [5]. Therefore, we can make a reliable estimate, $F / D=0.6-0.8$. This range includes the value from the $\mathrm{SU}(6)$ quark model $(F / D=2 / 3)$, and is slightly higher than that extracted from semi-leptonic decay rates of hyperons $(F / D \sim 0.57)$ [ [6]. Just as a comparison, we also show the $F / D$ ratio obtained from $i \gamma_{5}$ structure in figure 2. We find that the prediction highly depends on the choice of $\theta$, which prevents reliable estimate for the $F / D$ ratio.

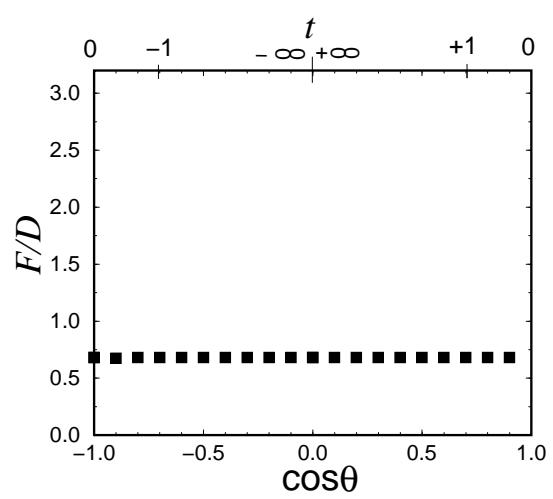

Figure 1. The $F / D$ ratio from $\gamma_{5} \sigma_{\mu \nu} q^{\mu} p^{\nu}$ structure against $\cos \theta$. Corresponding $t$ is also shown at the top of the figure.

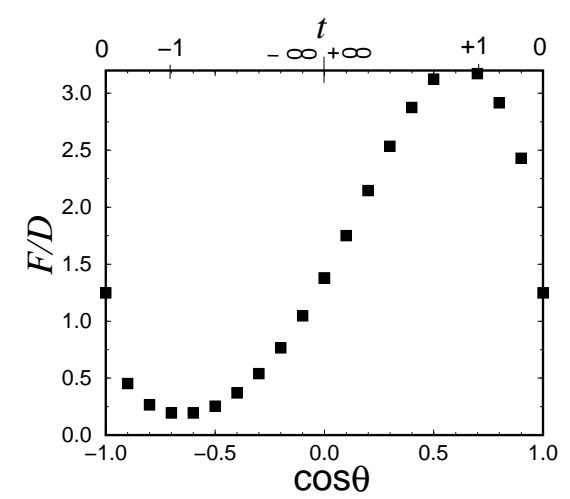

Figure 2. The $F / D$ ratio from $i \gamma_{5}$ structure against $\cos \theta$.

\section{THE $\pi \Lambda \Sigma$ COUPLING AND THE SU(3) BREAKING EFFECT}

We analyze the baryon-off-diagonal coupling $\pi \Lambda \Sigma$, and its $\mathrm{SU}(3)$ breaking effect using a projection method [ 7]. The advantages of this method are the followings: (1) The coupling constant is determined without referring to a priori effective Lagrangian, (2) baryon mass difference is taken into account properly, and (3) the single-pole terms and the continuum terms are well-defined in the correlation function. In this scheme, we calculate the projected correlation function defined as

$\Pi_{+}(q, p) \equiv \bar{u}_{\Sigma}(\boldsymbol{q}) \gamma_{0} \Pi(q, p) \gamma_{0} u_{\Lambda}(\boldsymbol{q}-\boldsymbol{p})$, 
where $\Pi(q, p)$ is given by Eq. (1) and $u(\boldsymbol{q})$ is the Dirac spinor for the plane wave with momentum $q$. In the phenomenological side, we obtain $g_{\pi \Lambda \Sigma}\left(\right.$ for $\left.\pi^{0} \Lambda \Sigma^{0}\right)$ as

$$
\begin{aligned}
& -\lambda_{\Lambda} \lambda_{\Sigma} \cdot g_{\pi \Lambda \Sigma} \cdot \bar{u}_{\Sigma}(\boldsymbol{q}) i \gamma_{5} u_{\Lambda}(\boldsymbol{q}-\boldsymbol{p}) \\
& \quad=\left.\bar{u}_{\Sigma}(\boldsymbol{q})\left(\not q-M_{\Sigma}\right) \Pi(q, p)\left(\not q-\not p-M_{\Lambda}\right) u_{\Lambda}(\boldsymbol{q}-\boldsymbol{p})\right|_{q^{2}=M_{\Sigma}^{2},(q-p)^{2}=M_{\Lambda}^{2}} \\
& \quad=\left(q_{0}-M_{\Sigma}\right)\left(q_{0}-E_{p}\right) \Pi_{+}(q),
\end{aligned}
$$

where $E_{p} \equiv \sqrt{M_{\Lambda}^{2}+\boldsymbol{p}^{2}}+\sqrt{m_{\pi}^{2}+\boldsymbol{p}^{2}}$. We use the dispersion relation with respect to $q_{0}$ for $\Pi_{+}^{\text {odd }} \equiv \frac{1}{2 q_{0}}\left[\Pi_{+}\left(q_{0}, \boldsymbol{q}\right)-\Pi_{+}\left(-q_{0}, \boldsymbol{q}\right)\right]$ to improve the sum rule. We note that though all Dirac structures in $\Pi(q, p)$ are mixed in $\Pi_{+}(q, p)$, $\Pi_{+}^{\text {odd }}(q, p)$ enhances the contribution from $\gamma_{5} \sigma_{\mu \nu} q^{\mu} p^{\nu}$ structure in $\Pi(q, p)$. We calculate the OPE up to dimension 7 , including terms proportional to the quark mass. We use the baryon mass sum rules to eliminate $\lambda_{B}$, and extract $g_{\pi \Lambda \Sigma}$ by operating $\left(1-M^{2} \frac{\partial}{\partial M^{2}}\right)$.

In figure 3, we show preliminary Borel plot from our result without continuum effect. We find that the Borel stability is obtained and we estimate $g_{\pi \Lambda \Sigma} \simeq 6-7$. Further, we include the $\mathrm{SU}(3)$ breaking effects via $m_{s} \neq m_{q}, \beta \neq\langle\bar{q} q\rangle, g\left\langle\bar{s} \sigma_{\mu \nu} G_{\mu \nu} s\right\rangle \neq g\left\langle\bar{q} \sigma_{\mu \nu} G_{\mu \nu} q\right\rangle$, $M_{B}(B=\Lambda, \Sigma) \neq M_{N}$, where $q$ denotes $\mathrm{u}$, d-quark. We show the Borel plot in figure 3 . From this figure, we see that the $\mathrm{SU}(3)$ breaking effect is small. More conclusive study including the continuum effects is in progress now [ 8].

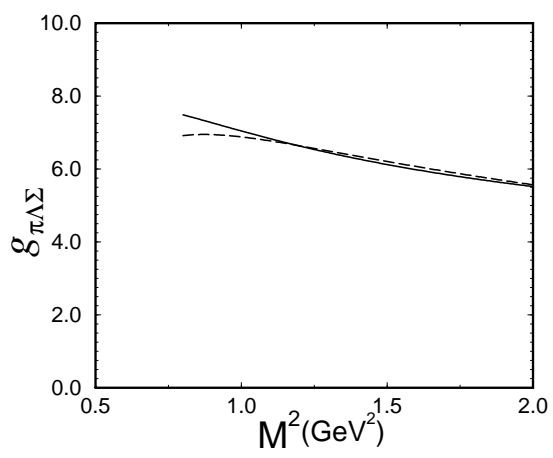

Figure 3. $g_{\pi \Lambda \Sigma}$ against the Borel mass $M^{2}$. The solid line denotes the $\mathrm{SU}(3)$ limit, the dashed line beyond $\mathrm{SU}(3)$ limit.

\section{REFERENCES}

1. M. C. Birse and B. Krippa, Phys. Lett. B 373 (1996) 9; Phys. Rev. C 54 (1996) 3240.

2. H. Shiomi and T. Hatsuda, Nucl. Phys. A 594 (1995) 294.

3. H. Kim, T. Doi, M. Oka and S. H. Lee, Nucl. Phys. A662 (2000) 371.

4. H. Kim, T. Doi, M. Oka and S. H. Lee, Nucl. Phys. A678 (2000) 295.

5. T. Doi , H. Kim and M. Oka, Phys. Rev. C 62 (2000) 055202.

6. P. G. Ratcliffe, Phys. Lett. B 365 (1996) 383.; hep-ph/9710458.

7. Y. Kondo and O. Morimatsu, Phys. Rev. C 66 (2002) 028201; nucl-th/0208018.

8. T. Doi, Y. Kondo and M. Oka, to be published. 